\title{
Nitrogen hydrides in the cold envelope of IRAS 16293-2422 $\star, \star \star$
}

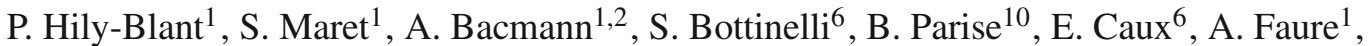 \\ E. A. Bergin ${ }^{25}$, G. A. Blake ${ }^{3}$, A. Castets ${ }^{1}$, C. Ceccarelli ${ }^{1}$, J. Cernicharo ${ }^{9}$, A. Coutens ${ }^{6}$, N. Crimier ${ }^{1,9}$, K. Demyk ${ }^{6}$, \\ C. Dominik ${ }^{12,13}$, M. Gerin ${ }^{28}$, P. Hennebelle ${ }^{28}$, T. Henning ${ }^{26}$, C. Kahane ${ }^{1}$, A. Klotz ${ }^{6}$, G. Melnick ${ }^{18}$, L. Pagani ${ }^{8}$, \\ P. Schilke ${ }^{10,20}$, C. Vastel $^{6}$, V. Wakelam ${ }^{2}$, A. Walters ${ }^{6}$, A. Baudry ${ }^{2}$, T. Bell ${ }^{3}$, M. Benedettini ${ }^{4}$, A. Boogert ${ }^{5}$, S. Cabrit $^{8}$, \\ P. Caselli ${ }^{7}$, C. Codella ${ }^{11}$, C. Comito ${ }^{10}$, P. Encrenaz ${ }^{8}$, E. Falgarone ${ }^{28}$, A. Fuente ${ }^{14}$, P. F. Goldsmith ${ }^{15}$, F. Helmich ${ }^{16}$,

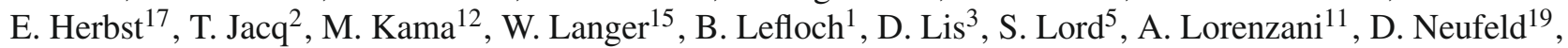 \\ B. Nisini ${ }^{24}$, S. Pacheco ${ }^{1}$, T. Phillips ${ }^{3}$, M. Salez ${ }^{8}$, P. Saraceno ${ }^{4}$, K. Schuster ${ }^{21}$, X. Tielens ${ }^{22}$, F. van der Tak ${ }^{16,27}$, \\ M. H. D. van der Wiel ${ }^{16,27}, \mathrm{~S}$. Viti ${ }^{23}$, F. Wyrowski ${ }^{10}$, and H. Yorke ${ }^{15}$
}

(Affiliations are available on page 5 of the online edition)

Received 22 June 2010 / Accepted 2 September 2010

\section{ABSTRACT}

\begin{abstract}
Nitrogen is the fifth most abundant element in the Universe, yet the gas-phase chemistry of N-bearing species remains poorly understood. Nitrogen hydrides are key molecules of nitrogen chemistry. Their abundance ratios place strong constraints on the production pathways and reaction rates of nitrogen-bearing molecules. We observed the class 0 protostar IRAS 16293-2422 with the heterodyne instrument HIFI, covering most of the frequency range from 0.48 to $1.78 \mathrm{THz}$ at high spectral resolution. The hyperfine structure of the amidogen radical o- $\mathrm{NH}_{2}$ is resolved and seen in absorption against the continuum of the protostar. Several transitions of ammonia from 1.2 to $1.8 \mathrm{THz}$ are also seen in absorption. These lines trace the low-density envelope of the protostar. Column densities and abundances are estimated for each hydride. We find that $\mathrm{NH}_{\mathrm{NH}}: \mathrm{NH}_{3} \approx 5: 1: 300$. Dark clouds chemical models predict steady-state abundances of $\mathrm{NH}_{2}$ and $\mathrm{NH}_{3}$ in reasonable agreement with the present observations, whilst that of $\mathrm{NH}$ is underpredicted by more than one order of magnitude, even using updated kinetic rates. Additional modelling of the nitrogen gas-phase chemistry in dark-cloud conditions is necessary before having recourse to heterogen processes.
\end{abstract}

Key words. ISM: abundances - ISM: general - astrochemistry

\section{Introduction}

Nitrogen is the fifth most abundant element in the Universe and is a fundamental component of molecules associated with life. Nitrogen-bearing molecules are routinely observed towards a wide variety of environments, from the diffuse interstellar medium (Liszt \& Lucas 2001) to pre-stellar cores (Bergin \& Tafalla 2007) and protoplanetary disks (Dutrey et al. 1997). Complex N-bearing molecules are also observed towards starforming regions (Herbst \& van Dishoeck 2009). The chemical network of nitrogen is apparently simple in that a small set of reactions is involved (Pineau des Forêts et al. 1990, hereafter PdF90). The chemistry of nitrogen has been modelled in various environments with moderate success, including typical dark cloud conditions (Millar et al. 1991; Le Bourlot 1991), shocks (PdF90), pre-stellar cores (Flower et al. 2006; Maret et al. 2006; Hily-Blant et al. 2010), and photo-dissociation regions (PDR) (Sternberg \& Dalgarno 1995). One major unknown is the total abundance, in dense and shielded environments, of gasphase nitrogen, the reservoir of which consist of $\mathrm{N}$ and/or $\mathrm{N}_{2}$. Because they are not directly observable, estimates of their abundances rely on observations of other N-bearing compounds and chemical modelling. Observational constraints of the dominant

* Herschel is an ESA space observatory with science instruments provided by European-led principal Investigator consortia and with important participation from NASA.

$\star \star$ Appendices (pages 6,7) are only available in electronic form at http://www . aanda. org chemical pathways of the nitrogen chemistry and their kinetic rates are thus crucial.

In this respect, nitrogen hydrides are of utmost importance since they are among the first neutral N-bearing molecules formed in an initially atomic gas dominated by hydrogen and helium. Ammonia was among the first interstellar molecules detected in emission towards the Galactic centre (Cheung et al. 1968). The lightest radical, imidogen $\mathrm{NH}$, was observed in absorption by Meyer \& Roth (1991) along the diffuse line of sight towards $\zeta$ Per. Gas-phase models were found to underestimate the abundance of $\mathrm{NH}$, and dust grains were then proposed to solve part of the discrepancy (Meyer \& Roth 1991; Wagenblast et al. 1993). Amidogen $\left(\mathrm{NH}_{2}\right)$ was observed in absorption by van Dishoeck et al. (1993) from dense gas in Sgr B2. This source was also targeted by Goicoechea et al. (2004) with ISO. The ratios of the three hydrides were found to be $\mathrm{NH}: \mathrm{NH}_{2}: \mathrm{NH}_{3} \approx 1: 10: 100$, incompatible with the dark cloud value $\mathrm{NH}_{3} / \mathrm{NH}_{2}<3$ predicted by Millar et al. (1991). Unfortunately, the modelling of the chemistry in Sgr B2 is difficult due to the complexity of the source, which probably incorporates shock dynamics. The $\mathrm{NH}: \mathrm{NH}_{2}: \mathrm{NH}_{3}$ ratios measured in Sgr B2 may thus not be representative of cold dark clouds, and the chemistry of nitrogen hydrides in these environments remains largely unexplored.

In this paper, we present HIFI observations of the submillimetre lines of $\mathrm{NH}_{2}$ and $\mathrm{NH}_{3}$ in absorption against the continuum of the class 0 protostar IRAS 16293-2422. Section 2 summarizes the observation strategy and data reduction. In Sect. 3 we derive the column densities of $\mathrm{NH}_{2}$ and $\mathrm{NH}_{3}$. Abundances of 
the three lightest nitrogen hydrides are estimated in Sect. 4, and compared to steady-state models using updated reaction rates.

\section{Observations and data reduction}

The solar-mass protostar IRAS 16293-2422 was observed with the HIFI instrument onboard the Herschel Space Observatory, as part of the HIFI guaranteed time key program CHESS (Ceccarelli et al. 2010). Full spectral coverage of bands 1a (480-560 GHz), 3b (858-961 GHz), 4a $(949-1061 \mathrm{GHz}), 5 \mathrm{a}$ $(1.12-1.24 \mathrm{THz})$, and $7 \mathrm{a}(1.70-1.79 \mathrm{THz})$ were performed on 2010 Mar. 1, 3, and 19, using the Spectral Scan DSB mode with optimization of the continuum. The Wide Band Spectrometre (WBS) was used as a backend, providing us with a spectral resolution of $1.1 \mathrm{MHz}$ over an instantaneous bandwidth of $4 \times 1 \mathrm{GHz}$. The targeted coordinates were $\alpha_{2000}=16^{\mathrm{h}} 32^{\mathrm{m}} 22^{\mathrm{s}} 75, \delta_{2000}=-$ $24^{\circ} 28^{\prime} 34.2^{\prime \prime}$. The two reference positions were situated approximately $3^{\prime}$ east and west of the source. The beam size is well approximated by $H P B W=21.5^{\prime \prime} / \nu_{\mathrm{THz}}$. For the analysis, intensities were then brought to a main-beam temperature scale using $F_{\text {eff }}=0.96$ and $B_{\text {eff }}=0.70$.

The data were processed using the standard HIFI pipeline HIPE 2.8 (Ott 2010) up to frequency and amplitude calibrations (level 2). For the SIS bands 1 to 5, a single local-oscillator-tuning spectrum consists of 4 sub-bands of $1 \mathrm{GHz}$ for each polarization. The $1 \mathrm{GHz}$ chunks for bands 1 to 5 are then exported as FITS files in the CLASS90/GILDAS format ${ }^{1}$ (Hily-Blant et al. 2005) for subsequent data reduction and analysis. Despuring and residual bandpass effect subtraction were performed in CLASS90 using generic spectral-survey tools developed in our group. Sideband deconvolution is computed with the minimization algorithm of Comito \& Schilke (2002) implemented into CLASS90. Line identification used the Weeds CLASS90-addon developed by Maret et al. (2010), which provides an efficient interface to the public CDMS and JPL spectroscopic databases (Müller et al. 2001; Pickett et al. 1998).

\section{Results}

Figure 1 shows the detection of the hyperfine structure (HFS) of the $N=0-1, J=\frac{1}{2}-\frac{3}{2}$, and $J=\frac{1}{2}-\frac{1}{2}$ transitions of amidogen in its ortho form, with their strongest components at the rest frequencies $952.578354 \mathrm{GHz}$ and $959.511716 \mathrm{GHz}$, respectively (Müller et al. 1999). The HFS is almost entirely resolved with an intensity ratio that clearly deviates from optically thin LTE excitation. The $3 \sigma$ noise levels are indicated in each panel. The para- $\mathrm{NH}_{2}$ line was not detected and a $5 \sigma$ upper limit to the main HFS component at $947.725 \mathrm{GHz}$ is $\int \tau \mathrm{d} v \leq 0.2 \mathrm{~km} \mathrm{~s}^{-1}$. Figure 2 shows several transitions of ammonia seen in absorption, from 1168.4 to $1763.8 \mathrm{GHz}$. The fundamental rotational transition at $572.6 \mathrm{GHz}$ was also detected in emission (see Fig. A.3) but because it contains both emission and absorption, it is not discussed in this paper.

All column densities are derived assuming a single excitation temperature $T_{\mathrm{ex}}$ for each molecule. The opacity of each HFS component and the excitation temperature are determined by simultaneously fitting all HFS components, constraining the opacities to scale with $A_{u l} g_{u}$. The fit is performed in CLASS90 by applying the HFS method to the continuum-subtracted spectra $T_{\mathrm{mb}}=\left[J_{v}\left(T_{\mathrm{ex}}\right)-\right.$ $\left.J_{v}\left(T_{\mathrm{CMB}}\right)-T_{\mathrm{C}}\right]\left(1-\mathrm{e}^{-\tau_{u l}}\right)$. The total column density reads

${ }^{1}$ http://www.iram.fr/IRAMFR/GILDAS

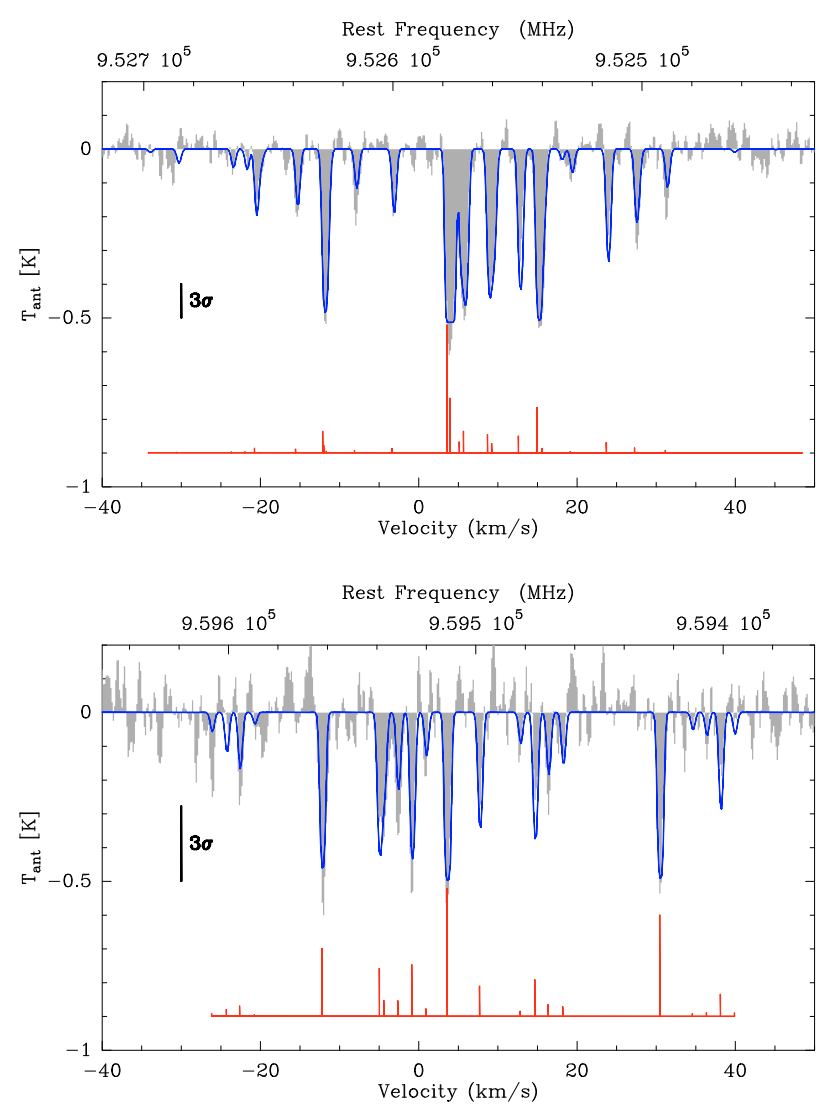

Fig. 1. Absorption spectra of o- $\mathrm{NH}_{2}$ at $952 \mathrm{GHz}$ and $959 \mathrm{GHz}$, with the HFS fit overlaid (line). The relative intensities in the optically thin LTE limit are indicated at the bottom.
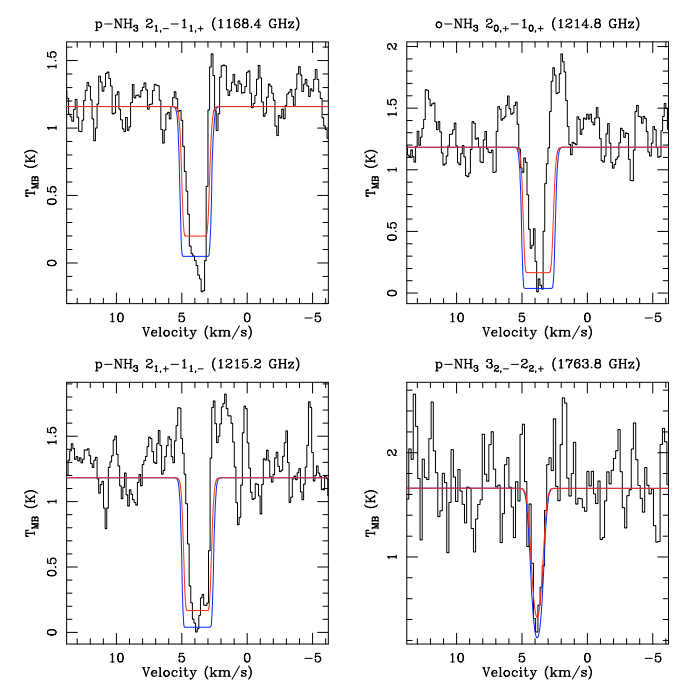

Fig. 2. $\mathrm{NH}_{3}$ absorption lines from 1.168 to $1.764 \mathrm{THz}$. LTE predictions are shown in red $\left(T_{\mathrm{ex}}=10 \mathrm{~K}, N\left(\mathrm{NH}_{3}\right)=3.5 \times 10^{15} \mathrm{~cm}^{-2}\right)$ and blue $\left(T_{\mathrm{ex}}=\right.$ $\left.8 \mathrm{~K}, N\left(\mathrm{NH}_{3}\right)=2 \times 10^{16} \mathrm{~cm}^{-2}\right)$.

$N_{\text {tot }}=\left(8 \pi v^{3} / c^{3}\right) Q /\left(A_{u l} g_{u}\right) \times \int \tau_{u l} \mathrm{~d} v \times \mathrm{e}^{E_{l} / k T_{\text {ex }}} /\left[1-\mathrm{e}^{-h v / k T_{\text {ex }}}\right]$, where $Q$ is the partition function at $T_{\mathrm{ex}}$. In deriving the excitation temperature, we assumed equal filling factors for the absorbing gas and the background continuum radiation. The SSB continuum intensity $T_{\mathrm{C}}$, needed to derive $T_{\mathrm{ex}}$, is estimated as half the median of each $1 \mathrm{GHz}$ chunk computed in line-free spectral windows prior to deconvolution, assuming equal gains in the two sidebands (see details in Appendix A). 
Table 1. Column densities of nitrogen hydrides towards IRAS 16293-2422.

\begin{tabular}{lcccccccccc}
\hline \hline Species & Transition & Component $^{a}$ & $\begin{array}{c}\text { Frequency } \\
\mathrm{GHz}\end{array}$ & $\begin{array}{c}\mathrm{HPBW} \\
\operatorname{arcsec}\end{array}$ & $\begin{array}{c}T_{\mathrm{C}, m b}{ }^{b} \\
\mathrm{~K}\end{array}$ & $\begin{array}{c}T_{l}^{c} \\
\mathrm{~K}\end{array}$ & $\begin{array}{c}\tau_{u l} \\
T_{\text {ex }} \\
\mathrm{K}\end{array}$ & $\begin{array}{c}F W H M^{d} \\
\mathrm{~km} \mathrm{~s}^{-1}\end{array}$ & $\begin{array}{c}N_{\text {tot }} \\
10^{14} \mathrm{~cm}^{-2}\end{array}$ \\
\hline $\mathrm{NH}^{e}$ & $0_{1} \rightarrow 1_{0}$ & $\frac{3}{2}, \frac{5}{2} \rightarrow \frac{1}{2}, \frac{3}{2}$ & 946.476 & 23 & 0.8 & $-3.8 \pm 0.6$ & $2.8 \pm 0.7$ & 9.5 & 0.60 & $2.20 \pm 0.80$ \\
$\mathrm{o}-\mathrm{NH}_{2}$ & $0_{00 \frac{1}{2}} \rightarrow 1_{11 \frac{3}{2}}$ & $\frac{3}{2}, \frac{5}{2} \rightarrow \frac{5}{2}, \frac{7}{2}$ & 952.578 & 23 & 0.9 & $-9.0 \pm 0.5$ & $12.8 \pm 0.7$ & 8.5 & 0.60 & $0.40 \pm 0.06$ \\
& $0_{00 \frac{1}{2}} \rightarrow 1_{11 \frac{1}{2}}$ & $\frac{3}{2}, \frac{5}{2} \rightarrow \frac{3}{2}, \frac{5}{2}$ & 959.512 & 22 & 0.9 & $-2.5 \pm 0.2$ & $4.9 \pm 0.7$ & 9.5 & 0.60 & $0.59 \pm 0.12$ \\
$\mathrm{p}^{-\mathrm{NH}_{3}}$ & $1-2$ & $(1,-)-(1,-)$ & 1168.453 & 18 & 1.2 & & $300-70$ & $8-10$ & 0.50 & $200-35$ \\
$\mathrm{o}_{3}-\mathrm{NH}_{3}$ & $1-2$ & $(0,+)-(0,+)$ & 1214.853 & 18 & 1.3 & & $470-130$ & $8-10$ & 0.50 & $200-35$ \\
$\mathrm{p}-\mathrm{NH}_{3}$ & $1-2$ & $(1,+)-(1,+)$ & 1215.246 & 18 & 1.3 & & $330-80$ & $8-10$ & 0.50 & $200-35$ \\
$\mathrm{p}-\mathrm{NH}_{3}$ & $2-3$ & $(2,-)-(2,-)$ & 1763.823 & 12 & 2.2 & & $2.0-1.4$ & $8-10$ & 0.50 & $200-35$ \\
\hline
\end{tabular}

Notes. ${ }^{(a)}$ For NH, the quantum numbers for the rotational transition $N_{J}$ are $\boldsymbol{F}_{1}=\boldsymbol{I}_{\mathrm{H}}+\boldsymbol{J}$ and $\boldsymbol{F}=\boldsymbol{I}_{\mathrm{N}}+\boldsymbol{F}_{1}$ (Klaus et al. 1997). For the $N_{K_{a} K_{c} J}$ rotational transition of $\mathrm{NH}_{2}$, the quantum numbers are $\boldsymbol{F}_{1}=\boldsymbol{I}_{\mathrm{N}}+\boldsymbol{J}$ and $\boldsymbol{F}=\boldsymbol{I}_{\mathrm{H}}+\boldsymbol{F}_{1}$ (Müller et al. 1999). In the case of ammonia, quantum numbers are given separately for $\boldsymbol{J}=\boldsymbol{N}+\boldsymbol{S}$ and $(K, \epsilon)$, where $\epsilon$ is the symmetry index (see Maret et al. 2009). For these lines, the frequency given is that of the brightest HF component. ${ }^{(b)}$ Single-sideband continuum in a $T_{\mathrm{mb}}$ scale. ${ }^{(c)} T_{l}=\tau_{u l}\left[J_{v}\left(T_{\mathrm{ex}}\right)-J_{v}\left(T_{\mathrm{CMB}}\right)-T_{\mathrm{C}, m b}\right]$ from the HFS fit. In the case of ammonia, see Sect. 3. ${ }^{(d)}$ A conservative uncertainty of $0.25 \mathrm{MHz}\left(0.08 \mathrm{~km} \mathrm{~s}^{-1}\right)$ imposed by the HIPE 2.8 pipeline was retained. (e) The integrated line opacity is calculated as $\int \tau \mathrm{d} v=1.06 F W H M \times \tau_{u l}{ }^{(d)}$ Bacmann et al. (2010).

It is found to increase with frequency and, for rest frequencies $v_{\mathrm{THz}}=0.492$ to $1.242 \mathrm{THz}, T_{\mathrm{C}}$ is well approximated by $T_{\mathrm{C}}\left(T_{\mathrm{A}}^{*},[\mathrm{~K}]\right)=1.10 v_{\mathrm{THz}}-0.42$ (see Fig. A.1). At higher frequencies, $T_{\mathrm{C}}$ is estimated from the deconvolution tool in the HIPE software, assuming equal sideband gains.

For ammonia, the hyperfine components in each lines are not resolved out by our observations, so the column density and excitation temperature were determined by simultaneously fitting the 4 lines shown in Fig. 2. The $1763.8 \mathrm{GHz}$ line has a well constrained centre-line opacity, yet the excitation temperature and column density remain degenerate. We therefore varied the excitation temperature between 5 to $15 \mathrm{~K}$, and adjusted accordingly the column density until the observed line profiles were reproduced. The line width was fixed to $0.5 \mathrm{~km} \mathrm{~s}^{-1}$. For $T_{\mathrm{ex}}>10 \mathrm{~K}$, the absorption lines at $1.11684,1.2148$, and $1.2152 \mathrm{THz}$ become significantly weaker than observed. In contrast, for $T_{\mathrm{ex}}<8 \mathrm{~K}$, the column density needed to reproduce the $1.7 \mathrm{THz}$ line becomes so large that the $1.2 \mathrm{THz}$ lines broaden significantly. The full set of constraints points towards $T_{\mathrm{ex}} \approx 8-10 \mathrm{~K}$ and $N_{\text {tot }} \approx 2.0 \times 10^{16}-3.5 \times 10^{15} \mathrm{~cm}^{-2}$. Figure 2 shows the results of the modelled lines corresponding to $T_{\mathrm{ex}}=8$ and $10 \mathrm{~K}$. The nondetection of the 1.7635 and $1.7636 \mathrm{THz}$ absorption lines (not shown in Fig. 2) is consistent with these models.

The results are summarized in Table 1 . The excitation temperature of the 952 and $959 \mathrm{GHz}$ transitions of $\mathrm{o}-\mathrm{NH}_{2}$ are significantly higher than $2.73 \mathrm{~K}$. The different values for each HFS most likely results from the LTE assumption not being entirely valid. However, $T_{\mathrm{ex}} \ll h v / k$, so that the corresponding uncertainties have negligible consequences on the column density. The thermalized ortho:para ratio is expected to be large at low temperatures $\left(\approx \mathrm{e}^{30.39 / T}\right.$, see Fig. A.2). Our two determinations of the $\mathrm{o}-\mathrm{NH}_{2}$ column density thus give an average for the total $\mathrm{NH}_{2}$ column density. The resulting $\mathrm{NH}_{2}$ and $\mathrm{NH}_{3}$ column densities are $0.44 \pm 0.07 \times 10^{14} \mathrm{~cm}^{-2}$ and $\approx 35-200 \times 10^{14} \mathrm{~cm}^{-2}$, respectively. The column density ratios are thus $\mathrm{NH} / \mathrm{NH}_{2}=5.0 \pm 1.2$ and $\mathrm{NH}_{3} / \mathrm{NH}_{2}=90-500$, or $\mathrm{NH}: \mathrm{NH}_{2}: \mathrm{NH}_{3} \approx 5: 1: 300$.

\section{Discussion}

From Table 1, the low excitation temperatures indicate that all lines are sub-thermally excited and therefore most likely trace regions with densities much lower than their critical densities, which are of the order of $10^{7} \mathrm{~cm}^{-3}$. The physical source model of Crimier et al. (2010) predicts densities lower than $\approx 10^{6} \mathrm{~cm}^{-3}$ for radii larger than $2400 \mathrm{AU}$ or $17^{\prime \prime}$ at a distance of $120 \mathrm{pc}$,
Table 2. Predicted steady-state fractional abundances of several nitrogen-bearing species in three different models.

\begin{tabular}{llllc}
\hline \hline Species & I & II & III & Observations \\
\hline $\mathrm{N}_{2} \mathrm{H}^{+a}$ & $100: 0$ & $90: 10$ & $90: 10$ & \\
$\mathrm{NH}_{4}^{+b}$ & $85: 2: 13$ & $85: 2: 13$ & $95: 2: 3$ & \\
$\mathrm{~N}_{2}$ & $1.0(-5)$ & $1.0(-5)$ & $1.0(-5)$ & - \\
$\mathrm{N}$ & $2.4(-7)$ & $2.5(-7)$ & $2.9(-7)$ & - \\
$\mathrm{NH}$ & $3.5(-11)$ & $9.5(-11)$ & $8.8(-11)$ & $2.0 \pm 1.0(-9)$ \\
$\mathrm{NH}_{2}$ & $1.9(-10)$ & $1.9(-10)$ & $1.3(-10)$ & $4.0 \pm 2.0(-10)$ \\
$\mathrm{NH}_{3}$ & $3.7(-8)$ & $3.7(-8)$ & $8.3(-8)$ & $0.3_{-0.1}^{+0.1}-2_{-0.8}^{+0.8}(-8)$ \\
$\mathrm{NH}_{2}: \mathrm{NH}_{2}: \mathrm{NH}_{3}$ & $0.2: 1: 190$ & $0.5: 1: 190$ & $0.7: 1: 640$ & $5: 1: 300$ \\
\hline
\end{tabular}

Notes. Numbers in parenthesis are powers of ten. Branching ratios (BR) for the dissociative recombination (DR) of $\mathrm{NH}_{2}^{+}$are $\mathrm{N}+\mathrm{H}_{2}(4 \%), \mathrm{NH}+$ $\mathrm{H}(39 \%)$ and $\mathrm{N}+2 \mathrm{H}(57 \%)$ (Thomas et al. 2005). ${ }^{(a)} \mathrm{BR}$ for the DR of $\mathrm{N}_{2} \mathrm{H}^{+}$into $\mathrm{N}_{2}+\mathrm{H}$ and $\mathrm{NH}+\mathrm{N}$, respectively (Molek et al. 2007; Adams et al. 2009). ${ }^{(b)} \mathrm{BR}$ for the $\mathrm{DR}$ of $\mathrm{NH}_{4}^{+}$into $\mathrm{NH}_{3}+\mathrm{H}, \mathrm{NH}_{2}+\mathrm{H}_{2}$, and $\mathrm{NH}_{2}+2 \mathrm{H}$, respectively (Öjekull et al. 2004). See additional details in Table B.1.

comparable to the $H P B W$ of the present observations, thus supporting the assumption of equal filling-factors for the absorbing gas and the continuum emission. At these radii, the modelled gas temperature is lower than $20 \mathrm{~K}$. The $\mathrm{NH}$ and $\mathrm{NH}_{2}$ linewidths are thus dominated by non-thermal broadening suggesting that turbulence has not been dissipated in the absorbing gas.

To place constraints on the average abundances in the foreground absorbing material, we need to measure the total $\mathrm{H}$ column density, which is not directly observable. The onedimensional density profile of Crimier et al. (2010) is extended to lower densities as $n_{\mathrm{H}_{2}}(r)=3 \times 10^{8}(r / 85 \mathrm{AU})^{-1.8}+10^{3} \mathrm{~cm}^{-3}$, to allow for a low-density envelope. The column density profile, convolved by the HIFI beam, is dominated by the lines-of-sight close to the centre. Considering only the gas in the regions with $n_{\mathrm{H}_{2}}<10^{6} \mathrm{~cm}^{-3}$, the column density is $N\left(\mathrm{H}_{2}\right) \approx 8 \times 10^{22} \mathrm{~cm}^{-2}$ or 80 magnitudes of visual extinction (assuming standard dust properties and that all $\mathrm{H}$ is molecular). Were the hydrides absorption to occur at densities lower than $10^{4}$ or $10^{5} \mathrm{~cm}^{-3}$, the total column density would decrease by a factor 3 . Therefore, in the following we estimate the abundances assuming $N\left(\mathrm{H}_{2}\right)=$ $5.5 \pm 2.5 \times 10^{22} \mathrm{~cm}^{-2}$. Results are summarized in Table 2 .

The column density ratios observed in the cold envelope of IRAS 16293-2422 differ from those derived by Goicoechea et al. (2004), who found NH: $\mathrm{NH}_{2}: \mathrm{NH}_{3}=1: 10: 100$ towards Sgr B2. These authors noted that these ratios are not consistent with typical dark cloud conditions but can be explained by shock 
chemistry. Though the $\mathrm{NH}_{3} / \mathrm{NH}_{2}$ ratios are similar in both the cold envelope and the $\mathrm{Sgr} \mathrm{B} 2$ region, the $\mathrm{NH} / \mathrm{NH}_{2}$ ratios are drastically different, suggesting that different chemistry is at work in the two sources. To study this, we have employed the Astrochem gas-phase chemical code of Maret $\&$ Bergin (in prep.) combined with modified versions of the osu. 09.2008 chemical network ${ }^{2}$. Typical physical conditions for a cold molecular cloud (gas temperature $T=10 \mathrm{~K}, n_{\mathrm{H}}=$ $2 \times 10^{4} \mathrm{~cm}^{-3}, A_{v}=10 \mathrm{mag}$ ) and a cosmic-ray ionization rate $\zeta=1.3 \times 10^{-17} \mathrm{~s}^{-1}$ were used. Higher extinctions would not modify the predicted steady-state abundances. In a similar way, higher densities would shorten the time to reach a steady-state but would not alter the corresponding abundances. The initial abundances are taken from Wakelam \& Herbst (2008) for which the $\mathrm{C} / \mathrm{O}$ gas-phase abundance ratio is 0.41 . The resulting timedependent fractional abundances of the nitrogen hydrides are shown on Fig. B.1. The steady-state is reached after a few $10^{6} \mathrm{yr}$, for which the $\mathrm{NH}: \mathrm{NH}_{2}: \mathrm{NH}_{3}$ abundance ratios are 0.2:1:190 (see Table 2). We note that the $\mathrm{NH}: \mathrm{NH}_{2}: \mathrm{NH}_{3}$ ratios are roughly constant for times $>10^{5} \mathrm{yr}$. If $\mathrm{NH}_{3} / \mathrm{NH}_{2}$ is consistent with the observations to within a factor of 2 , the steady-state $\mathrm{NH} / \mathrm{NH}_{2}$ ratio is too small by more than one order of magnitude because the abundance of $\mathrm{NH}$ is underpredicted by our model.

$\mathrm{NH}_{2}$ and $\mathrm{NH}_{3}$ are principally formed by the dissociative recombination (DR) of $\mathrm{NH}_{4}^{+}$(Le Bourlot 1991). The formation of $\mathrm{NH}$ is dominated by $\mathrm{NH}_{2}(\mathrm{O}, \mathrm{OH}) \mathrm{NH}$, unless the $\mathrm{NH}$ channel of the DR of $\mathrm{N}_{2} \mathrm{H}^{+}$has a non-zero branching ratio (BR). There are still disparate results for the BR of these DR reactions, and the most recent literature (see Table 2 and Florescu-Mitchell \& Mitchell 2006) suggest uncertainties of at least $10 \%$ in the BR. We conducted three model calculations, where we varied the BR by $10 \%$, to explore the effects on $\mathrm{NH}: \mathrm{NH}_{2}: \mathrm{NH}_{3}$. The results are summarized in Table 2 . The abundances vary at most by factors of a few. Noticeable is the increase in $n(\mathrm{NH})$ associated with the opening of the $\mathrm{NH}$ channel of the $\mathrm{DR}$ of $\mathrm{N}_{2} \mathrm{H}^{+}$. In any case, $\mathrm{NH}$ remains underabundant by almost two orders of magnitude. This deficit is reminiscent of the early results obtained in the diffuse medium that motivated the recourse to surface reactions. We recall that our models do not include freeze-out onto dust grains to enhance the role of gas-phase reactions. In the case of $\mathrm{NH}$ formation, however, the role of surface reactions is poorly constrained.

\section{Conclusions}

We have presented absorption spectra of the hyperfine structure of $\mathrm{NH}_{2}$ and several transitions of $\mathrm{NH}_{3}$. These lines emanate from the low density envelope of the protostar, at densities lower than typically $10^{6} \mathrm{~cm}^{-3}$, representative of typical dark cloud conditions. We have determined the column densities, as well as fractional abundances. We have found that $\mathrm{NH}: \mathrm{NH}_{2}: \mathrm{NH}_{3} \approx 5: 1: 300$. On the basis of on an updated chemical network, we have computed the steady-state abundances of amidogen and ammonia, which agree well with observed values. Imidogen is undepredicted by more than one order of magnitude, and $\mathrm{NH} / \mathrm{NH}_{2} \leq 1$. At this point, more observations (of e.g. ${ }^{15} \mathrm{~N}$ isotopologues) and modelling are clearly needed. Freeze-out and surface reactions, not included in our calculations, are potentially important. Before having recourse to dust surface processes, however, gas-phase chemistry has to be explored in far more details in studying e.g., the influence of the gas-phase $\mathrm{C} / \mathrm{O}$ ratio (Hily-Blant et al. 2010) or the effects of the $\mathrm{H}_{2}$ o:p ratio. The

\footnotetext{
2 http://www.physics.ohio-state. edu/ eric/research. html
}

consequences of the uncertainties in the rates of the dominant chemical paths (dissociative recombinations, neutral-neutral reactions at low temperature) shall also be explored in the process.

Acknowledgements. We thank the anonymous referee for useful comments. This paper benefitted from the CDMS and JPL databases. A. Faure is warmly acknowledged for his careful review of the reaction rates. HIFI has been designed and built by a consortium of institutes and university departments from across Europe, Canada and the United States under the leadership of SRON Netherlands Institute for Space Research, with major contributions from Germany, France and the US. Consortium members are: Canada: CSA, U.Waterloo; France: CESR, LAB, LERMA, IRAM; Germany: KOSMA, MPIfR, MPS; Ireland, NUI Maynooth; Italy: ASI, IFSI-INAF, Osservatorio Astrofisico di ArcetriINAF; Netherlands: SRON, TUD; Poland: CAMK, CBK; Spain: Observatorio Astronómico Nacional (IGN), Centro de Astrobiología (CSIC-INTA). Sweden: Chalmers University of Technology - MC2, RSS \& GARD; Onsala Space Observatory; Swedish National Space Board, Stockholm University - Stockholm Observatory; Switzerland: ETH Zurich, FHNW; USA: Caltech, JPL, NHSC.

\section{References}

Adams, N. G., Herd, C. R., Geoghegan, M., Smith, D., \& Canosa, A. 1991, J. Chem. Phys., 94, 4852

Adams, N. G., Molek, C. D., \& McLain, J. L. 2009, J. Phys. Conf. Ser., 192, 012004

Bacmann, A., Caux, E., Hily-Blant, P., Parise, B., \& Pagani, L. 2010, A\&A, 521, L42

Bergin, E. A., \& Tafalla, M. 2007, Ann. Rev. A\&A, 45, 339

Ceccarelli, C., Bacmann, A., Boogert, A., \& CHESS team. 2010, A\&A, 521, L22

Cheung, A. C., Rank, D. M., Townes, C. H., Thornton, D. D., \& Welch, W. J. 1968, Phys. Rev. Lett., 21, 1701

Comito, C., \& Schilke, P. 2002, A\&A, 395, 357

Crimier, N., Ceccarelli, C., Maret, S., et al. 2010, A\&A, 519, A65

Dutrey, A., Guilloteau, S., \& Bachiller, R. 1997, A\&A, 325, 758

Florescu-Mitchell, A. I., \& Mitchell, J. B. A. 2006, Phys. Rep., 430, 277

Flower, D. R., Pineau des Forêts, G., \& Walmsley, C. M. 2006, A\&A, 456, 215

Geppert, W. D., Thomas, R., Semaniak, J., et al. 2004, ApJ, 609, 459

Goicoechea, J. R., Rodríguez-Fernández, N. J., \& Cernicharo, J. 2004, ApJ, 600, 214

Herbst, E., \& van Dishoeck, E. F. 2009, Ann. Rev. A\&A, 47, 427

Hily-Blant, P., Pety, J., \& Guilloteau, S. 2005, CLASS evolution: I. Improved OTF support, Tech. rep., IRAM

Hily-Blant, P., Walmsley, M., Pineau des Forêts, G., \& Flower, D. 2010, A\&A, 513, A41

Klaus, T., Takano, S., \& Winnewisser, G. 1997, A\&A, 322, L1

Le Bourlot, J. 1991, A\&A, 242, 235

Liszt, H., \& Lucas, R. 2001, A\&A, 370, 576

Maret, S., Bergin, E. A., \& Lada, C. J. 2006, Nature, 442, 425

Maret, S., Faure, A., Scifoni, E., \& Wiesenfeld, L. 2009, MNRAS, 399, 425

Maret, S., Hily-Blant, P., Pety, J., Bardeau, S., \& Reynier, E. 2010, A\&A, submitted

Meyer, D. M., \& Roth, K. C. 1991, ApJ, 376, L49

Millar, T. J., Bennett, A., Rawlings, J. M. C., Brown, P. D., \& Charnley, S. B. 1991, A\&AS, 87, 585

Mitchell, B. 1990, Phys. Rep., 186, 215

Molek, C. D., McLain, J. L., Poterya, V., \& Adams, N. G. 2007, J. Phys. Chem. A, 111,6760

Müller, H. S. P., Klein, H., Belov, S. P., et al. 1999, J. Mol. Spectr., 195, 177

Müller, H. S. P., Thorwirth, S., Roth, D. A., \& Winnewisser, G. 2001, A\&A, 370, L49

Öjekull, J., Andersson, P. U., NåGåRd, M. B., et al. 2004, J. Chem. Phys., 120, 7391

Ott, S. 2010, in Astronomical Data Analysis Software and Systems XIX, ed. Y. Mizumoto, K.-I. Morita, \& M. Ohishi, ASP Conf. Ser.

Pickett, H. M., Poynter, I. R. L., Cohen, E. A., et al. 1998, J. Quant. Spect. Rad. Trans., 60, 883

Pineau des Forêts, G., Roueff, E., \& Flower, D. R. 1990, MNRAS, 244, 668

Sternberg, A., \& Dalgarno, A. 1995, ApJS, 99, 565

Talbi, D. 2009, J. Phys. Conf. Ser., 192, 012015

Thomas, R. D., Hellberg, F., Neau, A., et al. 2005, Phys. Rev. A, 71, 032711

van Dishoeck, E. F., Jansen, D. J., Schilke, P., \& Phillips, T. G. 1993, ApJ, 416, L83

Wagenblast, R., Williams, D. A., Millar, T. J., \& Nejad, L. A. M. 1993, MNRAS, 260,420

Wakelam, V., \& Herbst, E. 2008, ApJ, 680, 371 
1 Laboratoire d'Astrophysique de Grenoble, UMR 5571-CNRS, Université Joseph Fourier, Grenoble, France

2 Université de Bordeaux, Laboratoire d'Astrophysique de Bordeaux, CNRS/INSU, UMR 5804, Floirac, France

3 California Institute of Technology, Pasadena, USA

${ }_{4}$ INAF - Istituto di Fisica dello Spazio Interplanetario, Roma, Italy

5 Infared Processing and Analysis Center, Caltech, Pasadena, USA

${ }^{6}$ Centre d'Étude Spatiale des Rayonnements, Université Paul Sabatier, Toulouse 3, CNRS UMR 5187, Toulouse, France

7 School of Physics and Astronomy, University of Leeds, Leeds UK

8 LERMA and UMR 8112 du CNRS, Observatoire de Paris, 61 Av. de l'Observatoire, 75014 Paris, France

9 Centro de Astrobiología, CSIC-INTA, Madrid, Spain

10 Max-Planck-Institut für Radioastronomie, Bonn, Germany

11 INAF Osservatorio Astrofisico di Arcetri, Florence Italy

12 Astronomical Institute "Anton Pannekoek", University of Amsterdam, Amsterdam, The Netherlands

13 Department of Astrophysics/IMAPP, Radboud University Nijmegen, Nijmegen, The Netherlands

14 IGN Observatorio Astronómico Nacional, Alcalá de Henares, Spain
15 Jet Propulsion Laboratory, Caltech, Pasadena, CA 91109, USA

16 SRON Netherlands Institute for Space Research, Groningen, The Netherlands

17 Ohio State University, Columbus, OH, USA

18 Harvard-Smithsonian Center for Astrophysics, Cambridge MA, USA

19 Johns Hopkins University, Baltimore MD, USA

20 Physikalisches Institut, Universität zu Köln, Köln, Germany

21 Institut de RadioAstronomie Millimétrique, Grenoble, France

22 Leiden Observatory, Leiden University, Leiden, The Netherlands

23 Department of Physics and Astronomy, University College London, London, UK

${ }^{24}$ INAF - Osservatorio Astronomico di Roma, Monte Porzio Catone, Italy

25 Department of Astronomy, University of Michigan, Ann Arbor, USA

${ }^{26}$ Max-Planck-Institut für Astronomie, Heidelberg, Germany

27 Kapteyn Astronomical Institute, University of Groningen, The Netherlands

28 LERMA, UMR 8112-CNRS, Ecole Normale Supérieure et Observatoire de Paris, France 


\section{Appendix A: Determination of the column density}

Figure A.1 displays the double-sideband continuum intensity measured from 0.5 to $1.2 \mathrm{THz}$ with the double-sideband receivers of the HIFI instrument. The single-sideband continuum is then estimated assuming equal image and signal gains. The SSB continuum intensity was estimated following two independent methods. First, the median of the intensity in each $1 \mathrm{GHz}$ sub-band was computed. The resulting double-sideband (DSB) continuum level $T_{\mathrm{C}}$, sampled every $0.25 \mathrm{GHz}$ from $0.492 \mathrm{THz}$ to $1.242 \mathrm{THz}$, was found to increase linearly with the frequency (see Fig. A.1). The second method consisted in deconvolving the despured spectra prior to baseline subtraction, the result of which is the SSB continuum level. The DSB deconvolution was applied to the subset of spectra covering the spectral ranges of the lines considered in this paper. The DSB continuum level is twice the SSB to better than $10 \%$ in these intervals. From the first method, the antenna temperature scale SSB continuum level is well fitted by a 1 st order polynomial as $T_{\mathrm{C}}[\mathrm{K}]=1.10 v_{\mathrm{THz}}-0.42$, for rest frequencies $\nu_{\mathrm{THz}}$ ranging from 0.492 to $1.242 \mathrm{THz}$ (see Fig. A.1). The increase in $T_{\mathrm{C}}$ with frequency might be caused by the convolution of the dust temperature profile with the telescope beam. It may also trace the increase in the dust emissivity with frequency. At the higher frequencies of the ammonia lines in Band 7, the continuum level was estimated using the second method, in HIPE.

Figure A. 2 shows the o:p ratio for $\mathrm{NH}_{2}$ assuming equilibrium at a single temperature for all levels.

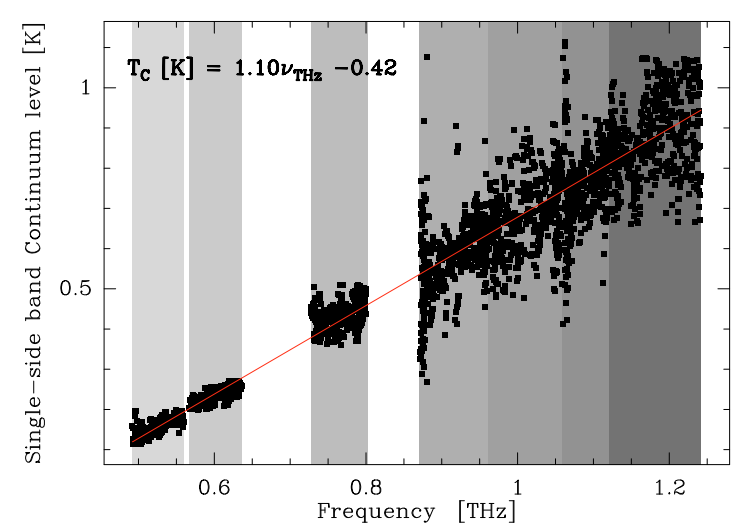

Fig. A.1. Double sideband continuum (in antenna temperature scale) level towards IRAS 16293-2422, from 0.5 to $1.2 \mathrm{THz}$. The straight line is the result of a linear fit. The observed HIFI bands are highlighted.

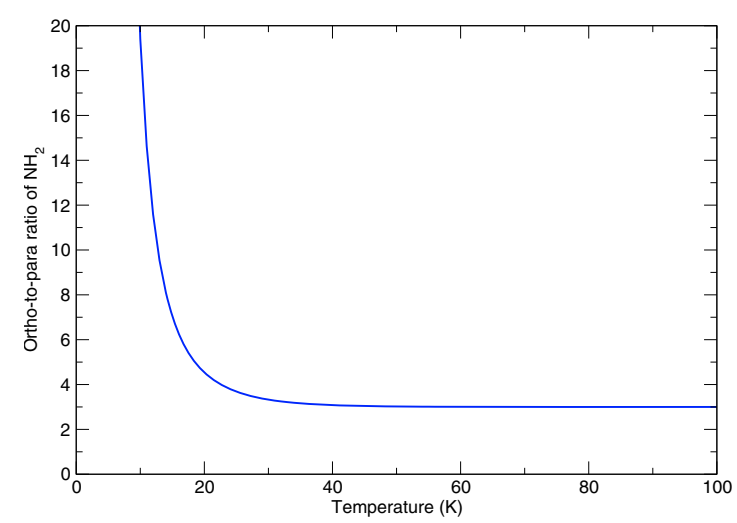

Fig. A.2. Thermalized ortho:para ratio for $\mathrm{NH}_{2}$.

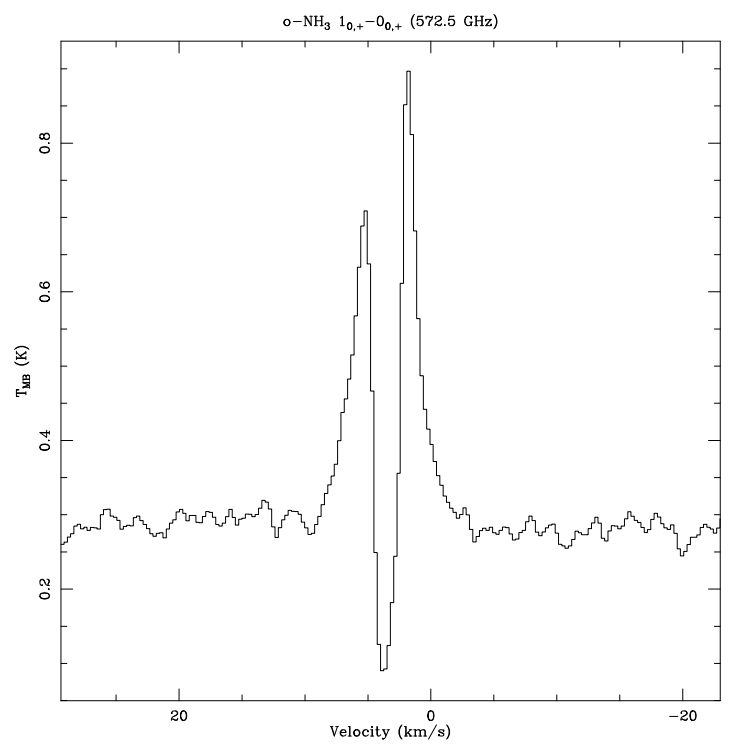

Fig. A.3. Emission line of the fundamental transition of $\mathrm{NH}_{3}$ at 572.6 GHz detected by HIFI towards IRAS 16293-2422.

\section{Appendix B: Chemical modelling}

Nitrogen chemistry starts with the formation of $\mathrm{NO}$ and $\mathrm{CN}$ by means of the reactions $N(\mathrm{OH}, \mathrm{NO}) \mathrm{H}$ and $N(\mathrm{CH}, \mathrm{H}) \mathrm{CN}$, which then lead to $\mathrm{N}_{2}$ (Hily-Blant et al. 2010). Once $\mathrm{N}_{2}$ is formed, it reacts with $\mathrm{He}^{+}$to form $\mathrm{N}^{+}$, which, by successive hydrogen abstractions, leads quickly to $\mathrm{NH}^{+}, \mathrm{NH}_{2}^{+}, \mathrm{NH}_{3}^{+}$, and $\mathrm{NH}_{4}^{+}$. The dissociative recombination (DR) of $\mathrm{NH}_{4}^{+}$is the dominant formation route for $\mathrm{NH}_{2}$ and $\mathrm{NH}_{3}$. The formation of $\mathrm{NH}$ is dominated by $\mathrm{NH}_{2}(\mathrm{O}, \mathrm{H}) \mathrm{NH}$, unless the $\mathrm{NH}$ channel of the DR of $\mathrm{N}_{2} \mathrm{H}^{+}$has a non-zero branching ratio (BR). Although DR branching ratios have been (re)measured recently for $\mathrm{N}_{2} \mathrm{H}^{+}, \mathrm{NH}_{2}^{+}$, and $\mathrm{NH}_{4}^{+}$, there are still disparate results and significant uncertainties. According to the most recent litterature (see Table 2 and Florescu-Mitchell \& Mitchell 2006), the BR of DR reactions are uncertain by at least $10 \%$. We conducted three model calculations, where we varied the BR by $10 \%$, to explore the effects on $\mathrm{NH}: \mathrm{NH}_{2}: \mathrm{NH}_{3}$. The results are summarized in Table 2 . The abundances vary at most by factors of a few. We note the increase in $n(\mathrm{NH})$, which is associated with the opening of the $\mathrm{NH}$ channel of the DR of $\mathrm{N}_{2} \mathrm{H}^{+}$. In any case, $\mathrm{NH}$ remains underabundant by almost two orders of magnitude. This deficit is reminiscent of the early results obtained in the diffuse medium that motivated the recourse to surface reactions. We recall that our models do not include freeze-out onto dust grains to ensure that the role of gas-phase reactions is enhanced. In the case of NH formation, however, the role of surface reactions is largely ill-constrained.

Following the above update of DR rates and branching ratios, we have adopted three different chemical models by varying the DR branching ratios of $\mathrm{N}_{2} \mathrm{H}^{+}$and $\mathrm{NH}_{4}^{+}$within $10 \%$, the typical experimental uncertainty. The DR of these two ions was indeed found to be the dominant formation routes of $\mathrm{NH}$ and $\mathrm{NH}_{2}$, respectively. The employed branching ratios are listed in Table B.1.

To model the steady-state abundances of the nitrogen hydrides, the osu.09.2008 network and rates were used. The osu.09.2008 network contains 13 elements, 449 species, and 4457 gas-phase reactions. We note that this version of the OSU database does not contain molecular anions or any depletion of gas-phase species. The network has been updated from a revision of the branching ratios and rate coefficients for the 
P. Hily-Blant et al.: Nitrogen hydrides in the envelope of IRAS 16293-2422

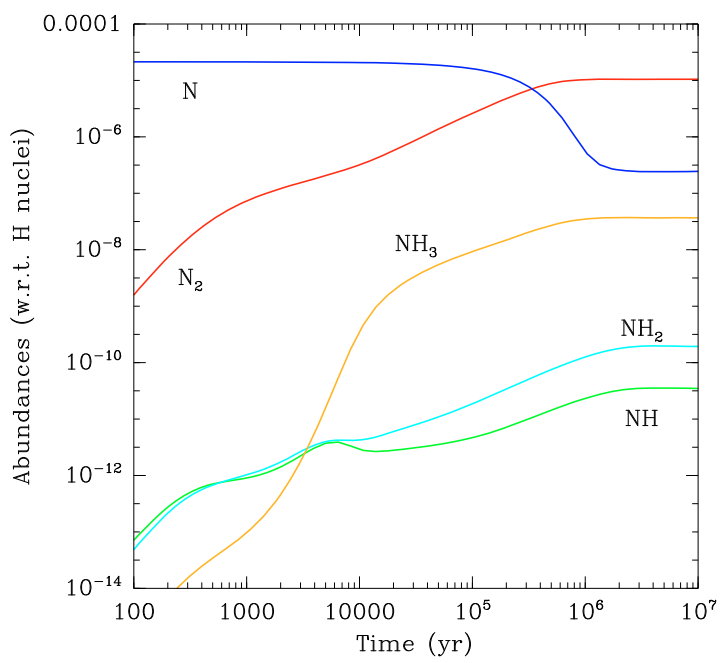

Fig. B.1. Predicted abundances for several N-bearing molecules as a function of time for model 1 ("standard", see text), for a density $n_{\mathrm{H}}=2 \times 10^{4} \mathrm{~cm}^{-3}$, a gas temperature $T_{\mathrm{kin}}=10 \mathrm{~K}$, and a total extinction $A_{\mathrm{V}}=10$ mag. Abundances are given with respect to $\mathrm{H}$ nuclei.

dissociative recombination (DR) of the nitrogen bearing cations $\mathrm{N}_{2} \mathrm{H}^{+}, \mathrm{NH}_{2}^{+}$, and $\mathrm{NH}_{4}^{+}$:

- The DR of $\mathrm{N}_{2} \mathrm{H}^{+}$has been determined experimentally using both flowing afterglow (FA, see Adams et al. 1991) and storage ring (SR) techniques (Geppert et al. 2004), leading to controversial results concerning the branching ratios (BR) of the two channels $\mathrm{N}_{2}+\mathrm{H}$ and $\mathrm{NH}+\mathrm{N}$. Thus, in contrast to the FA results that established the major product as $\mathrm{N}_{2}$ $+\mathrm{H}$ with a $\mathrm{BR} \approx 100 \%$, Geppert et al. (2004) found this channel to account for only $36 \%$ of the total reaction. The most recent FA and SR measurements (Molek et al. 2007; Adams et al. 2009), however, have confirmed the earlier FA results that the DR of $\mathrm{N}_{2} \mathrm{H}^{+}$should lead predominantly to $\mathrm{N}_{2}+\mathrm{H}$ with a $\mathrm{BR} \approx 90-100 \%$. This result is also supported by the $a b$ initio calculations of Talbi (2009). For the total rate
Table B.1. Dissociative recombination branching ratios employed in the present work.

\begin{tabular}{lcclrrr}
\hline \hline Ion & \multicolumn{2}{c}{ Total rate $^{a}$} & Products & \multicolumn{3}{c}{ Model } \\
& $\gamma$ & $\beta$ & & 1 & 2 & 3 \\
\hline $\mathrm{N}_{2} \mathrm{H}^{+b}$ & $1.00(-7)$ & -0.5 & $\mathrm{~N}_{2}+\mathrm{H}$ & $100 \%$ & $90 \%$ & $90 \%$ \\
& & & $\mathrm{NH}+\mathrm{H}$ & $0 \%$ & $10 \%$ & $10 \%$ \\
$\mathrm{NH}_{2}^{+c}$ & $3.00(-7)$ & -0.5 & $\mathrm{~N}+\mathrm{H}+\mathrm{H}$ & $57 \%$ & & \\
& & & $\mathrm{~N}+\mathrm{H}_{2}$ & $4 \%$ & & \\
& & & $\mathrm{NH}+\mathrm{H}$ & $39 \%$ & & \\
$\mathrm{NH}_{3}^{+}$ & $3.10(-7)$ & -0.5 & $\mathrm{NH}+\mathrm{H}+\mathrm{H}$ & $50 \%$ & & \\
& & & $\mathrm{NH}_{2}+\mathrm{H}$ & $50 \%$ & & \\
$\mathrm{NH}_{4}^{+d}$ & $9.40(-7)$ & -0.6 & $\mathrm{NH}_{3}+\mathrm{H}$ & $85 \%$ & $85 \%$ & $95 \%$ \\
& & & $\mathrm{NH}_{2}+\mathrm{H}_{2}$ & $2 \%$ & $2 \%$ & $2 \%$ \\
& & & $\mathrm{NH}_{2}+\mathrm{H}+\mathrm{H}$ & $13 \%$ & $13 \%$ & $3 \%$ \\
\hline
\end{tabular}

Notes. ${ }^{(a)}$ Reaction rates are written as $k=\gamma(T / 300)^{\beta}$ in $\mathrm{cm}^{3} \mathrm{~s}^{-1}$. ${ }^{(b)}$ Molek et al. (2007), ${ }^{(c)}$ Thomas et al. (2005), ${ }^{(d)}$ Öjekull et al. (2004).

coefficient, we adopted the (temperature dependent) expression of Geppert et al. (2004), as in the osu.09.2008 network.

- For the DR of $\mathrm{NH}_{2}^{+}$, the latest SR measurements are those of Thomas et al. (2005), who obtained the BR for $\mathrm{N}+\mathrm{H}_{2}$ $(4 \%), \mathrm{NH}+\mathrm{H}(39 \%)$, and $\mathrm{N}+2 \mathrm{H}(57 \%)$. For the rate coefficient, we adopted the expression recommended by Mitchell (1990), as in the osu.09.2008 network.

- Finally, the SR measurements of Öjekull et al. (2004) demonstrated that the $\mathrm{DR}$ of $\mathrm{NH}_{4}^{+}$is dominated by the product channels $\mathrm{NH}_{3}+\mathrm{H}(85 \%), \mathrm{NH}_{2}+2 \mathrm{H}(13 \%)$, and $\mathrm{NH}_{2}+\mathrm{H}_{2}(2 \%)$. For the total rate, we adopted the expression of Öjekull et al. (2004), which differs slightlty from the one recommended in the osu. 09.2008 network.

We note that for $\mathrm{NH}_{3}^{+}$there is to our knowledge neither measurements nor calculations available. We therefore adopted the rate and branching ratios recommended in the osu. 09.2008 network, corresponding to the two channels $\mathrm{NH}+2 \mathrm{H}(50 \%)$ and $\mathrm{NH}_{2}+\mathrm{H}(50 \%)$. 